\title{
Tying up loose ends
}

\author{
K. Yang $\cdot$ T. M. Connolly $\cdot$ R. Bergamaschi
}

Received: 18 November 2014/ Accepted: 13 February 2015/Published online: 24 February 2015

(C) Springer-Verlag Italia Srl 2015

\section{Dear Sir,}

Hemorrhoids are among the most common anorectal diseases seen in the USA and Europe. Hemorrhoidectomy has been long considered as the gold standard in the definitive treatment of hemorrhoids secondary to its low long-term recurrence rate. Historically, this has outweighed the severe postoperative pain and prolonged recovery. Several innovative techniques have been proposed as alternative modalities to address these concerns, while matching the low recurrence rates seen following hemorrhoidectomy. Among the alternative procedures is dearterialization, a non-excisional technique that has historically raised skepticism as to the anatomical rationale for ligating hemorrhoidal arteries, the usefulness of Doppler guidance, and most importantly, unknown long-term recurrence rate [1-3].

In the 20 years since dearterialization was first introduced, there have been a number of studies and randomized trials [4-7] evaluating its efficacy relative to preexisting surgical modalities. A recent systematic review [8] including 2904 patients from twenty-eight studies concluded that dearterialization is associated with less postoperative pain and is safe for patients with grade III hemorrhoids. In fact, in 2010, the UK National Institute for Health and Care Excellence described [9] dearterialization as an "efficacious alternative to conventional hemorrhoidectomy" with "no major safety concerns," citing level I evidence in its recommendation. Furthermore, follow-up data at 3 years post-dearterialization demonstrated

K. Yang $\cdot$ T. M. Connolly $\cdot$ R. Bergamaschi $(\bowtie)$ Division of Colon and Rectal Surgery, Health Science Center T18, State University of New York, Suite 046B, Stony Brook, NY 11794-8191, USA

e-mail: rcmbergamaschi@gmail.com comparable recurrence rates with fewer chronic complications as compared to hemorrhoidectomy [10].

Despite supporting evidence of the benefits of dearterialization, there continues to be a paucity of acknowledgement and discussion in the current literature. A recent review [11] on hemorrhoids published in New England Journal of Medicine, albeit otherwise comprehensive, fails to provide but one sentence under the "area of uncertainty" section discussing dearterialization. While the article states that more studies are needed to show the efficacy of dearterialization, it overlooks established evidence and recommendations provided within its own references [12], which likely reflects a lack of experience with THD in the USA. Nonetheless, there is a new large series of patients treated with THD in the USA published in this issue [13]. Although this study is retrospective, it shows that there is increasing attention to this procedure in that country.

This editorial is neither suggesting that the aforementioned studies are without flaws nor negating the need for larger randomized trials with superiority design and longer follow-up. Nevertheless, the value of dearterialization is often understated by narrative reviews and outdated guidelines. This should bring to light the ample data supporting dearterialization as an efficacious modality and tie up the loose ends of current literature on the surgical management of hemorrhoids.

Conflict of interest None.

\section{References}

1. Aigner F, Bodner G, Conrad F, Mbaka G, Kreczy A, Fritsch H (2004) The superior rectal artery and its branching pattern with regard to its clinical influence on ligation techniques for internal hemorrhoids. Am J Surg 187:102-108 
2. Gupta PJ, Kalaskar S, Taori S, Heda PS (2011) Doppler-guided hemorrhoidal artery ligation does not offer any advantage over suture ligation of grade 3 symptomatic hemorrhoids. Tech Coloproctol 15:439-444

3. Jongen J, Peleikis HG (2006) Doppler-guided hemorrhoidal artery ligation: an alternative to hemorrhoidectomy. Dis Colon Rectum 49:1082-1083

4. Morinaga K, Hasuda K, Ideda T (1995) A novel therapy for internal hemorrhoids: ligation of the hemorrhoidal artery with a newly devised instrument (Moricorn) in conjunction with a Doppler flow meter. Am J Gastroenterol 90:610-613

5. Elmer SE, Nygren JO, Lenander CE (2013) A randomized trial of transanal hemorrhoidal dearterialization with anopexy compared with open hemorrhoidectomy in the treatment of hemorrhoids. Dis Colon Rectum 56:484-490

6. Denoya PI, Fakhoury M, Chang K, Fakhoury J, Bergamaschi R (2013) Dearterialization with mucopexy versus haemorrhoidectomy for grade III or IV haemorrhoids: short-term results of a double-blind randomized controlled trial. Colorectal Dis 15:1281-1288

7. De Nardi P, Capretti G, Corsaro A, Staudacher C (2014) A prospective, randomized trial comparing the short- and long-term results of doppler-guided transanal hemorrhoid dearterialization with mucopexy versus excision hemorrhoidectomy for grade III hemorrhoids. Dis Colon Rectum 57:348-353

8. Pucher PH, Sodergren MH, Lord AC, Darzi A, Ziprin P (2013) Clinical outcome following Doppler-guided haemorrhoidal artery ligation: a systematic review. Colorectal Dis 15:e284-e294

9. National Institute for Health and Care Excellence. Interventional procedure guidance 342: haemorrhoidal artery ligation. http:// www.nice.org.uk/guidance/ipg342/resources/guidance-haemor rhoidal-artery-ligation-pdf. Accessed Oct 2014

10. Denoya P, Tam K, Bergamaschi R (2014) Hemorrhoidal dearterialization with mucopexy versus hemorrhoidectomy: 3-year follow-up assessment of a randomized control trial. Tech Coloproctol 18:1081-1085

11. Jacobs D (2014) Clinical Practice. Hemorrhoids. N Engl J Med 371:944-951

12. Altomare DF, Giuratrabocchetta S (2013) Conservative and surgical treatment of haemorrhoids. Nat Rev Gastroenterol Hepatol 10:513-521

13. LaBella GD, Main WPL, Hussain LR (2015) Evaluation of transanal hemorrhoidal dearterialization: a single surgeon experience. Tech Coloproctol. doi:10.1007/s10151-015-1269-6 